\title{
EXTENSIONS OF LOCALLY COMPACT ABELIAN GROUPS. II
}

\author{
BY \\ RONALD O. FULP AND PHILLIP A. GRIFFITH( $\left.{ }^{1}\right)$
}

\begin{abstract}
It is shown that the extension functor defined on the category $\mathscr{L}$ of locally compact abelian groups is right-exact. Actually $\mathrm{Ext}^{n}$ is shown to be zero for all $n \geqq 2$. Various applications are obtained which deal with the general problem as to when a locally compact abelian group is the direct product of a connected group and a totally disconnected group. One such result is that a locally compact abelian group $G$ has the property that every extension of $G$ by a connected group in $\mathscr{L}$ splits iff $G=(\boldsymbol{R} / \boldsymbol{Z})^{\sigma} \oplus \boldsymbol{R}^{n}$ for some cardinal $\sigma$ and positive integer $n$.
\end{abstract}

1. In a previous paper [3] we set up the homological theory needed for the development of an extension functor Ext for the category $\mathscr{L}$ of locally compact abelian groups. In that paper some of the properties of Ext were discovered and various applications were found. It was shown that Ext preserves exactness except possibly at the right end of the usual long exact sequence connecting Hom and Ext. In this paper we show that Ext is right-exact and in fact that $\operatorname{Ext}^{n}=0$ for $n \geqq 2$. Also further applications are found relating to the general problem as to when a locally compact abelian group splits into the direct product of a connected group and a totally disconnected one.

We refer the reader to [3] for pertinent comments regarding the historical development of our subject. Also see [3] regarding notation and terminology.

2. The functor Ext is right-exact. Our first theorem proves to be a useful reduction theorem. In some instances, it may be used to show that it is sufficient to work with compactly generated groups.

THEOREM 2.1. Suppose that $\phi: G \rightarrow H$ is a proper epimorphism in $\mathscr{L}$ and that $H$ is compactly generated. Then there is a compactly generated subgroup $L$ of $G$ such that $\phi(L)=H$. Moreover, $L$ can be chosen so that $G / L$ is discrete.

Proof. By Theorem 24.30 of Hewitt and Ross [5], $G=M \oplus R^{n}$ where $M$ contains some compact open subgroup $B$. Since $B \oplus R^{n}$ is open in $G, \phi\left(B \oplus R^{n}\right)$ is

Presented to the Society, January 24, 1969; received by the editors October 9, 1968 and, in revised form, March 20, 1970.

AMS 1968 subject classifications. Primary 2220; Secondary 2210.

Key words and phrases. Locally compact abelian groups, topological group extensions, right exactness of Ext, Ext ${ }^{n}=0$, split exact sequences, connected by totally disconnected, totally disconnected by connected.

(1) The research of the first author was supported in part by NASA Grant NGR 44-005-073 and a University Grant No. FRSP 6834. The second author was also partially supported by Grant GP-8750. 
open in $H$ and thus $H / \phi\left(B \oplus R^{n}\right)$ is discrete. Since $H / \phi\left(B \oplus R^{n}\right)$ is also compactly generated, it follows that $H / \phi\left(B \oplus R^{n}\right)$ is finitely generated. Note, however, that $\phi$ induces a proper epimorphism $\phi^{*}$ from $M / B$ onto $H / \phi\left(B \oplus R^{n}\right)$. Thus there exists a subgroup $B_{1}$ of $M$ such that $B \subseteq B_{1}, B_{1} / B$ is finitely generated, and $\phi^{*}$ maps $B_{1} / B$ onto $H / \phi\left(B \oplus R^{n}\right)$. Observe that $B_{1}$ is necessarily closed in $M$ since it contains the open subgroup $B$ of $M$ and thus is open in $M$. It follows that $H=\phi\left(B+R^{n}\right)$. Also $B_{1}$ is compactly generated since both $B$ and $B_{1} / B$ are compactly generated. Hence $L=B_{1}+R^{n}$ is compactly generated and $\phi(L)=H$. Clearly $G / L \cong M / B_{1}$ is discrete.

COROLlary 2.2. Suppose that $A$ is a group in $\mathscr{L}$ and that it is an extension of some discrete free subgroup $F$ of $A$ by a compactly generated group $C$ in $\mathscr{L}$. Then $A=F_{1}+$ $A_{1}$, where $A_{1}$ is compactly generated and $F_{1}$ is a subgroup of $F$ such that $F=F_{1} \oplus$ $\left(F \cap A_{1}\right)$ and $F \cap A_{1}$ has finite rank.

Proof. By Theorem 2.1, $A$ has a compactly generated subgroup $L$ such that $(L+F) / F=A / F$ and such that $A / L$ is discrete. Hence $L+F=A$. Since closed subgroups of compactly generated groups are again compactly generated, it follows that $F \cap L$ is finitely generated. Therefore $F=F_{1} \oplus F_{2}$ where $F_{2}$ has finite rank and $F \cap L \subseteq F_{2}$. Let $A_{1}=F_{2}+L$. Since $L$ is closed in $A$ and $A_{1} / L$ is closed in $A / L$, we have that $A_{1}$ is closed in $A$. Furthermore, $A_{1}$ is compactly generated since both $L$ and $A_{1} / L$ are compactly generated. It is routine to check that $F_{1} \cap A_{1}=0$ and that $A=F_{1}+A_{1}$. Since $A_{1}$ is necessarily $\sigma$-compact, it follows from Corollary 3.2 of [3] that $A=F_{1} \oplus A_{1}$. Finally, observe that $F \cap A_{1}=F_{2}$.

For notation and terminology regarding $n$-fold exact sequences see Mac Lane [8]. Our main deviation from Mac Lane is the basic assumption that all groups and morphisms are assumed only to be in $\mathscr{L}$ unless explicitly stated otherwise. Also, an $n$-fold extension of a group $A$ by a group $C$ is an exact sequence

$$
A \succ B_{1} \rightarrow B_{2} \rightarrow \cdots \rightarrow B_{n} \rightarrow C
$$

where each group of the sequence is a group in $\mathscr{L}$ and each map of the sequence is a proper morphism in $\mathscr{L}$.

LEMMA 2.3. Assume that

$$
S: A>\stackrel{\alpha}{\longrightarrow} B_{1} \stackrel{\beta_{1}}{\longrightarrow} \ldots \stackrel{\beta_{n-1}}{\longrightarrow} B_{n} \stackrel{\gamma}{\longrightarrow} C
$$

is an $n$-fold extension of $A$ by $C$ in $\mathscr{L}$ and that $B_{1}=M \oplus B_{1}^{\prime}$ where $M \subseteq \operatorname{Im} \alpha$. Then

(i) $A=N \oplus A^{\prime}$ where $\alpha(N)=M$,

(ii) $S^{\prime}: A^{\prime} \stackrel{\alpha^{\prime}}{\longrightarrow} B_{1}^{\prime} \stackrel{\beta_{1}^{\prime}}{\longrightarrow} B_{2} \rightarrow \cdots \stackrel{\beta_{n-1}}{\longrightarrow} B_{n} \stackrel{\gamma}{\rightarrow} C$ is an $n$-fold extension of $A^{\prime}$ by $C$ where $\alpha^{\prime}=\alpha \mid A^{\prime}$ and $\beta_{1}^{\prime}=\beta_{1} \mid B_{1}^{\prime}$, and

(iii) if $S^{\prime}$ is equivalent to the split $n$-fold extension of $A^{\prime}$ by $C$, then $S$ is equivalent to the split $n$-fold extension of $A$ by $C$. 
Proof. Parts (i) and (ii) are elementary. If $S^{\prime}$ is equivalent to the $n$-fold extension $A^{\prime} \stackrel{1_{A^{\prime}}}{\longrightarrow} A^{\prime} \rightarrow 0 \rightarrow \cdots \rightarrow 0 \rightarrow C \stackrel{1_{C}}{\rightarrow} C$, then one sees from Proposition 5.2 of Chapter III of [8] that $S$ is equivalent to the extension $A \succ A \rightarrow 0 \rightarrow \cdots \rightarrow 0 \rightarrow C \rightarrow C$. Part (iii) follows.

Each of the next four lemmas proves a special case of the theorem that $\operatorname{Ext}^{n}=0$ for all $n \geqq 2$. The theorem itself is pieced together from the lemmas and by the use of Theorem 2.1 to obtain the necessary reduction to the compactly generated case. We proceed without further discussion.

LEMMA 2.4. If $G$ is a group in $\mathscr{L}$ and $\operatorname{Ext}^{2}(C, G)=0$ for all compactly generated $C$ in $\mathscr{L}$, then $\operatorname{Ext}^{n}(C, G)=0$ for all compactly generated $C$ in $\mathscr{L}$ and all $n \geqq 2$.

Proof. Suppose that the conclusion holds for $n-1 \geqq 2$ and further that

$$
S: G \stackrel{\beta_{0}}{\longrightarrow} B_{1} \stackrel{\beta_{1}}{\longrightarrow} \cdots \stackrel{\beta_{n-1}}{\longrightarrow} B_{n} \stackrel{\beta_{n}}{\longrightarrow} C
$$

is an $n$-fold extension of $G$ by $C$ where $C$ is compactly generated. By Theorem 2.1, there is a compactly generated subgroup $B_{n}^{\prime}$ of $B_{n}$ such that $\beta_{n}\left(B_{n}^{\prime}\right)=C$. Let $B_{n-1}^{\prime}=$ $\beta_{n-1}^{-1}\left(B_{n}^{\prime} \cap \operatorname{Im} \beta_{n-1}\right)$. It is straightforward to show that $B_{n-1}^{\prime}$ is closed in $B_{n-1}$ and to show that

$$
S^{\prime}: G \stackrel{\beta_{0}}{\longrightarrow} B_{1} \longrightarrow \cdots \stackrel{\beta_{n-2}}{\longrightarrow} B_{n-1}^{\prime} \stackrel{\beta_{n-1}^{\prime}}{\longrightarrow} B_{n}^{\prime} \stackrel{\beta_{n}^{\prime}}{\longrightarrow} C
$$

is exact where $\beta_{n-1}^{\prime}=\beta_{n-1} \mid B_{n-1}^{\prime}$ and $\beta_{n}^{\prime}=\beta_{n} \mid B_{n}^{\prime}$. Actually, $S^{\prime}$ is proper exact since $B_{n}^{\prime}$ is open in $B_{n}$ and $B_{n-1}^{\prime}$ is open in $B_{n-1}$. Furthermore $S$ is equivalent to $S^{\prime}$. Also $S^{\prime}=S_{1} \circ S_{2}$ (Yoneda composite) where $S_{1}$ is the proper exact sequence

$$
G \succ B_{1} \rightarrow \cdots \rightarrow B_{n-2} \rightarrow B_{n-1}^{\prime} \rightarrow C_{1},
$$

where $C_{1}=\operatorname{Im} \beta_{n-1}^{\prime}$, and $S_{2}$ is the short exact sequence $C_{1} \succ B_{n}^{\prime} \stackrel{\beta_{n}^{\prime}}{\rightarrow} C$. Since $S_{1}$ represents an element in $\operatorname{Ext}^{n-1}\left(G, C_{1}\right), n-1 \geqq 2$, and $C_{1}$ is compactly generated ( $C_{1}$ is a closed subgroup of a compactly generated group), we have by the induction hypothesis that $S_{1}$ is equivalent to the split $n$-fold extension of $G$ by $C_{1}$. Since $S^{\prime}=S_{1} \circ S_{2}$, it follows that $S^{\prime}$, and hence $S$, is equivalent to the split $n$-fold extension of $G$ by $C$.

LeMmA 2.5. If $G$ has an injective resolution in $\mathscr{L}$, then $\operatorname{Ext}^{n}(A, G)=0$ for all $n \geqq 2$.

Proof. The lemma follows from the fact that if $G$ has an injective resolution, then it has a short injective resolution.

COROLlARY 2.6. If $F$ is a discrete free group of finite rank, then $\operatorname{Ext}^{n}(A, F)=0$ for all $A$ in $\mathscr{L}$ and $n \geqq 2$.

LEMMA 2.7. If $P$ is a projective in $\mathscr{L}$ and $C$ is a compactly generated group in $\mathscr{L}$, then $\operatorname{Ext}^{n}(C, P)=0$ for all $n \geqq 2$. 
Proof. By Lemma 2.4, it suffices to show that $\operatorname{Ext}^{2}(C, P)=0$ for all compactly generated groups $C$ of $\mathscr{L}$. Since vector groups are injective in $\mathscr{L}$ it follows from Theorem 3.3 of [9] that we may assume $P$ is free. Suppose $S: P \stackrel{\alpha}{\rightarrow} A \stackrel{\beta}{\rightarrow} B \stackrel{\gamma}{\rightarrow} C$ represents an arbitrary element of $\operatorname{Ext}^{2}(P, C)$. We show that $S$ is equivalent to the split extension. From the proof of Lemma 2.4, we see that $S$ is equivalent to a 2-fold extension $S^{\prime}: P \stackrel{\alpha^{\prime}}{\longrightarrow} A^{\prime} \stackrel{\beta^{\prime}}{\longrightarrow} B^{\prime} \stackrel{\gamma^{\prime}}{\rightarrow} C$ where $B^{\prime}$ is compactly generated. Thus we may assume that $B$ is already compactly generated. It follows that $\operatorname{Im} \beta$ is also compactly generated. By Corollary 2.2, we have that $A=A_{1} \oplus F_{1}$ and $P=P_{1} \oplus N$ where $\alpha\left(P_{1}\right)=F_{1}$ and $N$ has finite rank. By Lemma 2.3, $S_{1}: N \stackrel{\alpha_{1}}{\longrightarrow} A_{1} \stackrel{\beta_{1}}{\longrightarrow} B \stackrel{\gamma}{\rightarrow} C$ is exact where $\alpha_{1}=\alpha \mid N$ and $\beta_{1}=\beta \mid A_{1}$. Moreover, by Corollary 2.6, $S_{1}$ is equivalent to the split 2-fold extension of $N$ by $C$. Thus by Lemma 2.3, $S$ is equivalent to the split 2-fold extension of $P$ by $C$.

LEMMA 2.8. If $N$ is a group in $\mathscr{L}$ having no small subgroups, then $\operatorname{Ext}^{n}(G, N)=0$ for all groups $G$ in $\mathscr{L}$ and all $n \geqq 2$.

Proof. First suppose that $N$ is projective. By Theorem 2.9 of [3], there is a proper exact sequence $P_{1} \succ P \oplus K \rightarrow G$ where $P_{1}$ and $P$ are projectives in $\mathscr{L}$ and $K$ is compactly generated. Therefore we obtain the exact sequence

$$
\operatorname{Ext}^{n-1}\left(P_{1}, N\right) \rightarrow \operatorname{Ext}^{n}(G, N) \rightarrow \operatorname{Ext}^{n}(P \oplus K, N)
$$

We have that $\operatorname{Ext}^{n-1}\left(P_{1}, N\right)=0$ and $\operatorname{Ext}^{n}(P \oplus K, N) \cong \operatorname{Ext}^{n}(K, N)$ since $P_{1}$ and $P$ are projective. However, $\operatorname{Ext}^{n}(K, N)=0$ for all $n \geqq 2$ by Lemma 2.7. Thus $\operatorname{Ext}^{n}(G, N)=0$ for projective $N$ and $G$ any group of $\mathscr{L}$. Note, however, that if $N$ is a group which has no small subgroups, then it follows from Theorem 3.6 of [9] that $N$ has a projective resolution. Thus it easily follows that $\operatorname{Ext}^{n}(G, N)=0$ for each $G$ in $\mathscr{L}$ and each $N$ having no small subgroups.

THEOREM 2.9. If $G$ and $H$ are groups in $\mathscr{L}$, then $\operatorname{Ext}^{n}(G, H)=0$ for all $n \geqq 2$.

Proof. By Theorem 2.9 of [3], there is a short proper exact sequence

$$
H \longmapsto I \oplus N \rightarrow J
$$

where $I$ and $J$ are injectives and $N$ has no small subgroups. We therefore obtain the short exact sequence

$$
\operatorname{Ext}^{n-1}(G, J) \rightarrow \operatorname{Ext}^{n}(G, H) \rightarrow \operatorname{Ext}^{n}(G, I \oplus N) .
$$

But $\operatorname{Ext}^{n-1}(G, J)=0$ and $\operatorname{Ext}^{n}(G, I \oplus N) \cong \operatorname{Ext}^{n}(G, N)$ since $I$ and $J$ are injective. Furthermore $\operatorname{Ext}^{n}(G, N)=0$ for all $n \geqq 2$ by Lemma 2.8. Thus $\operatorname{Ext}^{n}(G, H)=0$ for all $n \geqq 2$.

We are now able to improve Theorem 2.14 of [3]. 
COROLlaRY 2.10. If $G$ is a group in $\mathscr{L}$ and $A \longrightarrow B \rightarrow C$ is a proper short exact sequence in $\mathscr{L}$, then the sequences of discrete abelian groups

$$
\begin{aligned}
\operatorname{Hom}(G, A) \succ \operatorname{Hom}(G, B) & \rightarrow \operatorname{Hom}(G, C) \\
& \rightarrow \operatorname{Ext}(G, A) \rightarrow \operatorname{Ext}(G, B) \rightarrow \operatorname{Ext}(G, C), \\
\operatorname{Hom}(C, G) \succ \operatorname{Hom}(B, G) & \rightarrow \operatorname{Hom}(A, G) \\
& \rightarrow \operatorname{Ext}(C, G) \rightarrow \operatorname{Ext}(B, G) \rightarrow \operatorname{Ext}(A, G)
\end{aligned}
$$

are exact.

This completes the proof of the main result of the paper. Our next and final section of the paper exhibits how our main results may be applied to various splitting problems in $\mathscr{L}$.

3. Splitting problems in $\mathscr{L}$. As has already been indicated, we are concerned with the problem as to when a group is the direct product of a connected group and a totally disconnected one. If a group $G$ is compact, then it admits such a decomposition if and only if its Pontrjagin dual $G^{\wedge}$ is the direct sum of a torsion group and a torsion free group. Thus for compact groups our problem is equivalent to the "mixed group" problem of discrete group theory. In [4] Harrison has developed a theory to handle those discrete groups which are, in a natural sense, those groups which are as "mixed as possible". These are the so called co-torsion groups. In [4] a (reduced) discrete group $H$ is called a co-torsion group if and only if Ext $(F, H)=0$ for all discrete torsion free $F$. An analogous development in the category $\mathscr{L}$ might be concerned with the parallel situation replacing the "torsion" concept with that of "connectedness". Actually we consider four distinct modifications of the co-torsion concept defined above.

Our first problem would be to describe the "co-connected" groups. This was actually done in [3]. We record the result for completeness.

THEOREM 3.1. A locally compact abelian group $G$ has the property that $\operatorname{Ext}(X, G)$ $=0$ for all totally disconnected $X$ in $\mathscr{L}$ if and only if $G=D \oplus T^{\sigma} \oplus R^{n}$ for some discrete divisible group $D$.

CoRollary 3.2. A connected group $G$ in $\mathscr{L}$ splits out of every group in $\mathscr{L}$ which contains it as its identity component if and only if it is of the form $T^{\sigma} \oplus R^{n}$.

Our next theorem describes the "co-totally disconnected" groups.

TheOREM 3.3. A locally compact abelian group $G$ has the property that $\operatorname{Ext}(C, G)$ $=0$ for all connected $C$ in $\mathscr{L}$ if and only if $G=T^{\sigma} \oplus R^{n}$.

Proof. The sufficiency follows from Theorem 3.2 of Moskowitz [9]. Therefore suppose that $G$ is in $\mathscr{L}$ and that $\operatorname{Ext}(C, G)=0$ for all connected $C$ in $\mathscr{L}$.

We first show that $G$ is connected. Assume that $G$ is not connected. By Theorem 24.30 of [5] $G=M \oplus R^{n}$ where $M$ contains some compact open subgroup. Since 
$G$ is not connected, it follows that there exists a nonzero totally disconnected homomorphic image of $G$ which, in turn, has a nonzero discrete homomorphic image. Let $A$ denote any discrete homomorphic image of $G$. Since Ext is rightexact and $\operatorname{Ext}(C, G)=0$ for all connected $C$ in $\mathscr{L}$, it follows that $\operatorname{Ext}(C, A)=0$ for all connected $C$ in $\mathscr{L}$. If $A$ were divisible it would follow from Theorem 3.1 that Ext $(X, A)=0$ for all totally disconnected $X$ in $\mathscr{L}$ and thus that $A$ is an injective of $\mathscr{L}$. The latter statement is contrary to Theorem 3.2 of Moskowitz [9]; thus $A$ is not divisible. It follows that $A$, and hence $G$, has a nonzero finite homomorphic image $F$. As with $A$, one sees that Ext $(C, F)=0$ for all connected $C$ of $\mathscr{L}$. But $F$ can be embedded in $T^{m}$ for some positive integer $m$ and thus $\operatorname{Ext}\left(T^{m} / F, F\right)=0$. It follows that $F$ is a direct summand of $T^{m}$ contrary to the finiteness of $F$. Thus $G$ is connected.

By Theorem 3.6 of [9], $G$ has an injective resolution $G \longleftrightarrow I \rightarrow J$. Since injectives are necessarily connected, $J$ is connected. Thus $G$ is a direct summand of $I$. Hence $G$ is injective in $\mathscr{L}$ and $G=T^{\sigma} \oplus R^{m}$ by Theorem 3.2 of [9].

The following corollary improves Moskowitz's Theorem 3.2 [9].

Corollary 3.4. $G$ is injective in $\mathscr{L}$ if and only if $\operatorname{Ext}(C, G)=0$ for all compact connected $C$ in $\mathscr{L}$.

Our last two theorems are variations of the latter two.

THeOREM 3.5. A locally compact abelian group $G$ has the property that $\operatorname{Ext}(G, X)$ $=0$ for all totally disconnected $X$ in $\mathscr{L}$ if and only if $G=\sum_{\sigma} Z \oplus R^{m}$.

Proof. The sufficiency follows from Theorem 3.3 of Moskowitz [9].

To prove the necessity, observe that $\operatorname{Ext}\left(C, G^{\wedge}\right)=0$ for all compact connected $C$ (see Theorem 2.12 of [3]). By Corollary 3.4, $G^{\wedge}$ is injective in $\mathscr{L}$ and $G^{\wedge}=$ $T^{\sigma} \oplus R^{n}$. Thus $G=\sum_{\sigma} Z \oplus R^{n}$ as required.

Our final theorem takes care of the only obvious remaining possibility.

THEOREM 3.6. A locally compact abelian group $G$ has the property that Ext $(G, C)=0$ for all connected $C$ of $\mathscr{L}$ if and only if $G=R^{n} \oplus M$ where $M$ contains a compact open subgroup having a co-torsion dual.

Proof. Let $G$ denote any locally compact abelian group. Then $G=M \oplus R^{n}$ where $M$ contains some compact open subgroup $K$. Since $K \longmapsto M \rightarrow M / K$ is a proper short exact sequence, then the sequence

$$
\operatorname{Ext}(M / K, C) \rightarrow \operatorname{Ext}(M, C) \rightarrow \operatorname{Ext}(K, C)
$$

is exact for all $C$ in $\mathscr{L}$. Note, however, that if $C$ is connected and $\mathscr{F}(C)$ denotes $C$ stripped of its topology, then by Proposition 2.17 of [3], we have $\operatorname{Ext}(M / K, C) \cong$ $\operatorname{Ext}_{z}(M / K, \mathscr{F}(C))=0$ (recall that $C$, being connected, is divisible). Thus $\operatorname{Ext}(M, C) \cong \operatorname{Ext}(K, C)$ for all connected $C$. It follows that $\operatorname{Ext}(M, C)=0$ for all connected $C$ if and only if $\operatorname{Ext}(K, C)=0$ for all connected $C$. But $\operatorname{Ext}(K, C)=0$ 
for all connected $C$ if and only if $\operatorname{Ext}\left(J, K^{\wedge}\right)=0$ for all discrete torsion free $J$, i.e. if and only if $K^{\wedge}$ is co-torsion.

We really have proven slightly more than the theorem states. The following is a corollary of the proof of the latter theorem.

COROLLARY 3.7. If $M$ is a locally compact abelian group which contains a compact open subgroup, then the following statements are equivalent:

(i) some compact open subgroup of $M$ has a co-torsion dual, and

(ii) every compact open subgroup of $M$ has a co-torsion dual.

\section{REFERENCES}

1. R. Baer, Erweiterung von Gruppen und ihren Isomorphismen, Math. Z. 38 (1934), 375-416.

2. S. Eilenberg and S. Mac Lane, Group extensions and homology, Ann. of Math. (2) 43 (1942), 757-831. MR 4, 88.

3. R. Fulp and P. Griffith, Extensions of locally compact abelian groups. I, Trans. Amer. Math. Soc. 154 (1970), 341-356.

4. D. K. Harrison, Infinite abelian groups and homological methods, Ann. of Math. (2) 69 (1959), 366-391. MR 21 \#3481.

5. E. Hewitt and K. Ross, Abstract harmonic analysis. Vol. I: Structure of topological groups. Integration theory, group representations, Die Grundlehren der math. Wissenschaften, Band 115, Academic Press, New York and Springer-Verlag, Berlin, 1963. MR 28 \#158.

6. G. Hochschild, Group extensions of Lie groups. I, Ann. of Math. (2) 54 (1951), 96-109. MR 13, 12.

7. — Group extensions of Lie groups. II, Ann. of Math. (2) 54 (1951), 537-551. MR 13, 318.

8. S. Mac Lane, Homology, Die Grundlehren der math. Wissenschaften, Band 114, Academic Press, New York and Springer-Verlag, Berlin, 1963. MR 28 \#122.

9. M. Moskowitz, Homological algebra in locally compact abelian groups, Trans. Amer. Math. Soc. 127 (1967), 361-404. MR 35 \#5861.

UNIVERSITY OF HOUSTON, Houston, TeXas 77004 